\title{
A Comparative Study of Malaria Diagnosis Using Rapid Diagnostic Tests (RDT) and Microscopy in Fever Patients in East and West Kupang District, Indonesia during the COVID- I 9 Pandemic
}

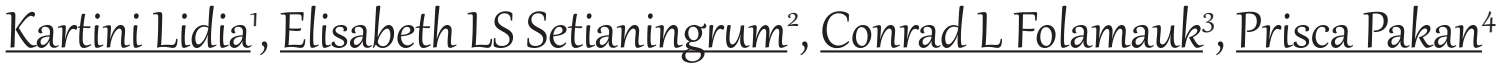 \\ ${ }^{1}$ Department of Pharmacology, Medical Faculty, University of Nusa Cendana, Indonesia. \\ ${ }^{2}$ Department of Clinical Pathology, Medical Faculty, University of Nusa Cendana, Indonesia. \\ ${ }^{3}$ Department of Tropical Medicine, Medical Faculty, University of Nusa Cendana, Indonesia. \\ ${ }^{4}$ Department of Microbiology, Medical Faculty, University of Nusa Cendana, Indonesia. \\ DOI: https://doi.org/10.24321/0019.5138.202126
}

\section{I $\quad \mathbf{N} \quad \mathbf{F} \quad \mathbf{O}$}

\section{Corresponding Author:}

Kartini Lidia, Department of Pharmacology, Medical Faculty, University of Nusa Cendana, Indonesia.

E-mail Id:

kartini_lidia@staf.undana.ac.id

Orcid Id:

https://orcid.org/0000-0003-0436-2239

How to cite this article:

Lidia K, Setianingrum ELS, Folamauk CL, Pakan P. A Comparative Study of Malaria Diagnosis Using Rapid Diagnostic Tests (RDT) and Microscopy in Fever Patients in East and West Kupang District, Indonesia during the COVID-19 Pandemic. J Commun Dis. 2021; 53(2): 57-61.

Date of Submission: 2021-03-12

Date of Acceptance: 2021-06-05

\section{$\begin{array}{lllllllllll}\mathbf{A} & \mathbf{B} & \mathbf{S} & \mathbf{T} & \mathbf{R} & \mathbf{A} & \mathbf{C} & \mathbf{T}\end{array}$}

Introduction: Malaria is a parasitic infectious disease that is still a world health problem, especially in tropical countries including Indonesia. Itis still a major cause of death in both adults and children. The majority of cases occur in the African region (93\%), followed by Southeast Asia including Indonesia (3.4\%), and the East Mediterranean region (2.1\%). The number of deaths due to malaria globally in 2018 reached 405,000, a decrease compared to416,000 in 2017 and 585,000 in 2010. In the era of theCOVID-19 pandemic.

Aim: This study is useful to provide a descriptive picture of the number of malaria casesthat presented with fever, which is also a common symptom of COVID-19.

Methods: This research is an interventional descriptive study with the aim of comparing the number of malaria cases with fever symptoms diagnosed through microscopic examination and Rapid Diagnostic Test (RDT) at the Batakte Health Centre located in West Kupang (Group 1) and at the Naibonat Health Centre (Group 2) located in East Kupang. The total sample in this study were 128 people with fever symptoms. In each group, the malaria blood smear was examined and RDT was carried out.

Result: The Result of the study revealedthat there were no malaria cases found in both Batakte Health Center and the Naibonat Health Center during the COVID-19 pandemic period.

Conclusion: There is no significant difference between the Batakte and Naibonat Health centres in terms of malaria diagnosis using both microscopy and RDT.

Keywords: Malaria, RDT, Microscopic, Kupang 


\section{Introduction}

Malaria is a parasitic infectious disease that is still a world health problem, especially in tropical countries including Indonesia. Malaria is still a major cause of death in both adults and children. According to the 2019 World Health Organization (WHO) malaria report, the estimated number of malaria cases in the world in 2018 had reached 228 million. The majority of cases occurred in the African region (93\%), followed by Southeast Asia including Indonesia (3.4\%), andthe East Mediterranean region (2.1\%). The incidence of malaria decreased globally between 2010 and 2018, from 71 to 51 cases per 1000 population at risk. The number of deaths due to malaria globally in 2018 reached 405,000, a decrease as compared to 416,000 in 2017 and 585,000 in $2010 . .^{1,2,3}$

Children under 5 years of age are the most vulnerable to malaria. In 2018, child deaths from malaria accounted for $67 \%$ of deaths from malaria worldwide. Data from the Indonesian Ministry of Health regarding the achievements of District/City Elimination of Malaria and Annual Parasite Incidence (API) indicated less than 1 death per province in 2019. There are 3 provinces where all districts and cities have achieved malaria elimination, namely Jakarta, Bali and East Java. The province of East Nusa Tenggara is one of the 5 provinces where there are no districts/cities that have achieved elimination of malaria. The five provinces are Papua, West Papua, Maluku, North Maluku, and East Nusa Tenggara. ${ }^{4}$

Since WHO declared COVID-19 a pandemic on March 11,2020 , the Indonesian government has made various efforts such as social distancing, physical distancing, selfquarantine, and has takenlarge-scale social restrictions measures. This affects the prevention and control of other infectious diseases, including malaria. The government has targetedtheelimination of malaria by 2024 in as many as 405 districts/cities. The 2020-2024 period is an important and decisive period in the effort to achieve a Malaria-Free Indonesia in 2030. Efforts to achieve the National Malaria Elimination target in 2030 in Indonesia, preceded by the stages of achieving a malaria-free area at the provincial level, subsequently, after all districts/cities in Indonesia have achieved malaria-free areas. ${ }^{5,6}$ The province of East Nusa Tenggara is targeting the elimination of malaria by 2023, of course, preceded by the achievement of malaria elimination at the district/city level, including Kupang Regency.

In the times of COVID-19 pandemic, data from the Indonesian Ministry of Health inJune 2020 regarding the Distribution of Endemic Malaria in Districts/Cities also affected by COVID-19, indicated a decrease in the number of positive cases of malaria between January 2019 and May 2020 by $54 \%$ and a decrease in positive cases of malaria by $57 \%$. On the other hand, most malaria-endemic areas have been affected by COVID-19, including East Nusa Tenggara. ${ }^{2,6}$ This fact should not be an obstacle in achieving the national malaria elimination target in 2030, especially since COVID-19 has several symptoms that are also clinically shared by malaria, such as fever, headaches, and muscle aches. ${ }^{5,6,7,8}$ Patients with confirmed malaria do not rule out the possibility that the patient may also suffer from COVID-19 as well as patients with suspected or confirmed COVID-19 who live in malaria-endemic areas, should also undergo malaria diagnostic tests without seeing symptoms. ${ }^{8,9}$ Therefore, it is necessary to increase awareness of an increase in malaria cases, especially in malaria-endemic areas during the COVID-19 pandemic era. The gold standard for diagnosis of malaria according to WHO is a microscopic examination using a thick and thin malaria blood smear. The duration of microscopic examination to get the results is approximately $20-60$ minutes. ${ }^{10,11,12}$ In thisera of the COVID-19 pandemic, malaria-endemic areas could provide malaria therapy based on RDT results to reduce the length of time patients have to wait for the results of malaria microscopic examination in health facilities. ${ }^{8}$

\section{Methods}

This is ainterventional descriptive studyandwas conducted from 1 July to 30 September 2020at the BatakteHealth Centre located in West Kupang (Group 1) and at the NaibonatHealth Centre (Group 2) located in East Kupang. The inclusion criteria for study include all the patients admitted to both Health Centres with fever symptoms (axillary temperature greater than $37.5^{\circ} \mathrm{C}$ )from 1 July to 30 September 2020, also all the respondents need to sign an informed consent. While the exclusion criteria include patients who were presented with fever symptoms (axillary temperature greater than $37.5^{\circ} \mathrm{C}$ ) but refused to participate in this study.

The total sample in this study were 128 people with fever symptoms (axillary temperature greater than $37.5^{\circ} \mathrm{C}$ ) subsequently divided into 64 people per group. In each group, the malaria blood smear was examined and the Rapid Diagnostic Test (RDT) was carried out to diagnose malaria. Thus, the research sample consisted of 128 slides of malaria blood smear and 128 results of the RDT of malaria. The sample comprised 64 people from PuskesmasBatakte representing Puskesmas located in West Kupang and 64 from PuskesmasNaibonat representing Puskesmas located in Kupang Timur. During the COVID-19 pandemic, there was a very significant decrease in the number of daily visits to health centres, which before the pandemic could range from 50 patients to 5-10 patients per day only during the pandemic now.

The instruments used in this study include information sheets for respondents and informed consent sheets, questionnaires, and forms of malaria examination results using RDT and microscopy. 
This study has received ethical approval from the Health Research Ethics Commission of the Faculty of Medicine, University of Nusa Cendana.

\section{Results}

\section{Demographic Characteristics of the Subjects at Naibonat Health Centre}

$38.64 \%$ of the respondents were male and $61.36 \%$ were female. $11.37 \%$ of the study subjects belonged to the age group of less than 1 year old, $29.55 \%$ belonged to the $2-5$ years old age group, $25 \%$ belonged to the age group of 6-17 years old, $20.46 \%$ belonged to the $18-35$ years old age group, $6.81 \%$ belonged to the age group of $36-55$ years old, $6.81 \%$ belonged to the age group of over 56 years old (Table 1 ).

Table I.Basic Characteristics of Research Subjects at the Naibonat Health Centre

\begin{tabular}{|c|c|}
\hline Characteristics & $N=44$ \\
\hline \multicolumn{2}{|c|}{ Gender, n (\%) } \\
\hline Male & $17(38.64)$ \\
\hline Female & $27(61.36)$ \\
\hline \multicolumn{2}{|c|}{ Age Groups (years) n (\%) } \\
\hline$\leq 1$ & $5(11.37)$ \\
\hline $2-5$ & $13(29.55)$ \\
\hline $6-17$ & $11(25)$ \\
\hline $18-35$ & $9(20.46)$ \\
\hline $36-55$ & $3(6.81)$ \\
\hline$\geq 56$ & $3(6.81)$ \\
\hline \multicolumn{2}{|c|}{ Body Temperature } \\
\hline Median (range), 으 & $38.5(37.5-39.0)$ \\
\hline
\end{tabular}

Table 2.BasicCharacteristics of Research Subjects at the Batakte Health Centre

\begin{tabular}{|c|c|}
\hline Characteristics & $N=86$ \\
\hline \multicolumn{2}{|c|}{ Gender n (\%) } \\
\hline Male & $22(25.58)$ \\
\hline Female & $64(74.42)$ \\
\hline \multicolumn{2}{|c|}{ Age Groups (years) n (\%) } \\
\hline$\leq 1$ & $9(10.46)$ \\
\hline $2-5$ & 27 (31.39) \\
\hline $6-17$ & $3(3.49)$ \\
\hline $18-35$ & 41 (47.67) \\
\hline $36-55$ & $4(4.65)$ \\
\hline$\geq 56$ & $2(2.34)$ \\
\hline \multicolumn{2}{|c|}{ Body Temperature } \\
\hline Median (range), 으 & 37.8 (36.5-39.0) \\
\hline
\end{tabular}

\section{Characteristicsof the Subjects Location atthe Batakte Health Centre}

As can be seen from Table 2, 25.58\% of the research subjects were male, and $74.42 \%$ were female. The basic characteristics of research subjects based on the age group, namely $10.46 \%$ of the participants belonged to the age group of less than equal to 1 year old,31.39\% belonged to the $2-5$ yearsold age group, $3.49 \%$ belonged to the age group of $6-17$ years old, $47.67 \%$ belonged to the age group of $18-35$ years old, $4.65 \%$ belonged to the age group of $36-55$ years, and $2.34 \%$ belonged to the age group of over 56 years.

\section{Microscopic Examination}

Thick and thin blood smearswere prepared at Naibonat Health Centre and Batakte Health Centre and were subsequently cross-checked at the Regional Health Laboratory, Province of East Nusa Tenggara. The results obtained after microscopic examination of smears, from bothhealth centres, were found to be negative for malaria. No change was found in the results after beingcross-checked at the Regional Health Laboratory, Province of East Nusa Tenggara.

\section{RDT Malaria Examination}

Apart from carrying out a microscopic examination of the subjects, a malaria Rapid Diagnostic Test (RDT) was also conductedunder the Carestaat brand. All the subjects showed negative results for malaria RDT. This means that there wereno malaria parasite antigens in all the research samples. The diagnostic target antigen can be specific to one Plasmodiumspecies or may include 4 plasmodium species. In this study, the RDT used did not detect the histidinerich protein 2 (HRP2) antigen from $P$. falciparum, parasite lactate dehydrogenase ( $\mathrm{p}-\mathrm{LDH})$, and aldolase produced by asexual or sexual forms of $P$. falciparum parasites, $P$. vivax, $P$. ovale, and P. malaria.

\section{Comparison of Results of Microscopic Examination and Rapid Diagnostic Test (RDT)}

When the results of the microscopic examination were compared with the results of the RDT, it did not show a significant difference between the Batakte and NaibonatHealth centres. The comparisonindicated that no malaria parasites were found either microscopically or through detection of parasite antigen via RDT.

\section{Discussion}

In this study, out of the 128 slides examined, none were positive microscopically or immunochromatographically using the malaria RDT. This study also compared microscopic examination and examination of RDT for malaria and found no significant difference in the examination results between the microscopic malaria diagnostic method and 
the immunochromatographic method using malaria rapid diagnostic test, in fever patients at Batakte Health Centre, West Kupang compared with the Naibonat Health Centre, East Kupang.

Microscopic examination of blood smears is still the gold standard for the diagnosis of malaria. , $8,9,10,12$ Preparations for malaria testing should be made when a patient has a fever to increase the likelihood of finding parasites.Blood samples should be taken before anti-malarial drugs are given so that parasites can be found if the patient has malaria. ${ }^{12}$ Blood samples weretaken from the tip of the ring finger for adult patients, while in infants, it was taken from the toe. Prior to the blood draw, an aseptic procedure is performed on the patient's fingertips. Microscopic diagnosis has a number of limitations. Among them, this examination requires a good quality microscope and power source as well as an expert and experienced microscopist. This examination also takes time, between 20-60 minutes. The quality of the smear affects the results of the examination. Variations in staining and the methods used to collect and treat blood samples may affect the interpretation of the preparations. The results of this examination are also influenced by the density of parasites. Routine microscopy cannot reliably detect very low parasitemia (5-10 parasites/ $\mu \mathrm{L}$ ). ${ }^{10,11}$

RDT is a tool that detects malaria antigen in small blood samples by immunochromatographic tests. Immunochromatographic tests are based on the capture of parasite antigens from peripheral blood using monoclonal or polyclonal antibodies against parasitic antigens. The antigen used as a diagnostic target can be specific to one plasmodium species, or may include 4 malaria parasites in humans. ${ }^{11,13}$ Currently immunochromatography tests can detect histidine-rich protein 2 (HRP2) from $P$. falciparum, parasite lactate dehydrogenase $(p-L D H)$, and aldolase produced by the asexual or sexual forms of the parasites $P$. falciparum, $P$. vivax, $P$. ovale, and $P$. malaria. ${ }^{11,13}$ RDT has several drawbacks, which include false positives and false negatives in some cases. False-positive results occur due to cross-reaction with rheumatoid factors in the blood. A rare false-negative result can be caused by a deletion or mutation of the hrp-2 gene. Another drawback of RDT is that it is unable to calculate the density of parasitemia, and its ability is less than optimal at low parasitemia. The quality of the RDT diagnostic tool is heavily correlated by the transportation and storage of the diagnostic tool. High humidity and temperature can quickly destroy reagents.

Similar research should continue to be carried out, especially in malaria-endemic areas, during the COVID-19 pandemic in order to achieve the 2030 malaria elimination target by continuing to carry out malaria microscopic examinations for public health centres or by using RDTs for health centres that do not have microscopy or that require examination with faster results for reduced waiting times in health facilities. Every health centre in malaria-endemic areas in East Nusa Tenggara Province must have sufficient RDT logistics because malaria screening is mandatory in the era of the COVID-19 pandemic, especially because the symptoms of malaria are almost the same as the symptoms of COVID-19.

\section{Conclusion}

There is no significant difference between the Batakte and Naibonat Health centres. In fact, it indicated that no malaria parasites were found either microscopically or through detection of parasite antigen via RDT.

\section{Acknowledgement}

This study was fully funded and supported by the University of Nusa Cendana, Indonesia.

\section{Conflict of Interest: None \\ References}

1. World Health Organization[Internet]. World Malaria Report 2019. 2019. WHO, Geneva, Switzerland. Available from: https://www.who.int/publications/i/ item/9789241565721

2. Ministry of Health of the Republic of Indonesia. 2019. Achievements of Malaria Elimination by Province in 2019. Ministry of Health, Republic of Indonesia, Jakarta.

3. The NTT Provincial Health Office. 2020. Final data for the 2019 Malaria Program. Provincial Health Office of NTT, Kupang.

4. Ministry of Health Republic of Indonesia. 2020 Guidelines for the Prevention and Control of Corona Virus Disease (Covid-19). Director General of Disease Prevention and Control, Ministry of Health, Republic of Indonesia, Jakarta.

5. East Nusa Tenggara Provincial Health Office. 2019. Malaria report for Kupang Regency 2019. NTT Provincial Health Office, Kupang.

6. Directorate General of Disease Prevention and Control, Ministry of Health of the Republic of Indonesia, 2017. Handbook of Malaria Case Management.

7. Ministry of Health of the Republic of Indonesia. 2020. Malaria Service Protocol during the Covid-19 pandemic. Director General of Disease Prevention and Control, Ministry of Health, Republic of Indonesia, Jakarta.

8. World Health Organization [Internet]. Tailoring malaria interventions in the COVID-19 response. 2020. WHO, Geneva, Switzerland. Available from: https://www.who. int/docs/default-source/documents/publications/gmp/ tailoring-malaria-interventions-covid-19.pdf

9. Kakkilaya BS. Rapid Diagnosis of Malaria. Labmed. 2003;34:602-8. [Google Scholar] 
10. Murray CK, Gasser RA, Magill AJ, Miller RS. Update on Rapid Diagnostic Testing for Malaria. Clin Microbiol Rev. 2008;21(1):97-110. [PubMed] [Google Scholar]

11. Ministry of Health Republic of Indonesia. 2017. Technical Guidelines for Malaria Examination, Directorate of Prevention of Vector-borne and Zoonotic Disease Control, Directorate General of Disease Prevention and Control, Ministry of Health, Republic of Indonesia, Jakarta.

12. Ministry of Health of the Republic of Indonesia. 2013. Basic Health Research 2018. Health Research and Development Agency of the Ministry of Health of the Republic of Indonesia, Jakarta.

13. Budi S. Prevalence of Clinical Malaria and Positive Plasmodium spp. Based on the Mass Blood Survey in West Southeast Maluku Regency.Aspirator. 2010;2(1):410. 